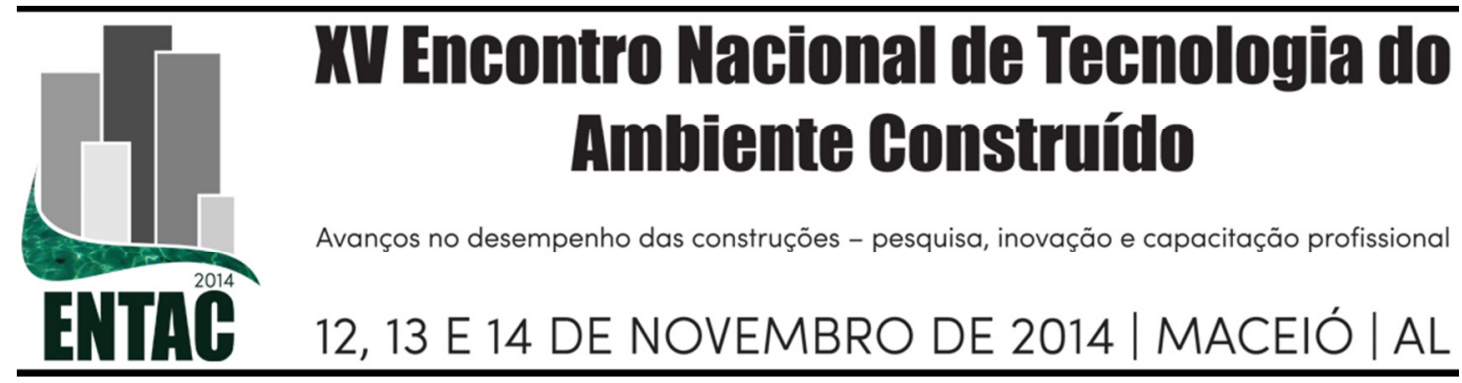

\title{
INFLUÊNCIA DE DIFERENTES CONFIGURAÇÕES URBANAS NO CONFORTO TÉRMICO EM CÂNIONS URBANOS DE CAMPINAS, SP - ESTUDOS DE CAMPO E SIMULAÇÕES COMPUTACIONAIS
}

\author{
ABREU-HARBICH, Loyde V. (1); SAMPAIO, Victor Hugo P. (2); LABAKI, \\ Lucila C. (3), MATZARAKIS, Andreas (4) \\ (1) Universidade Estadual de Campinas, e-mail: loydeabreu@gmail.com (2) Universidade Estadual de \\ Campinas, e-mail: victorhpsamp@gmail.com, (3) Universidade Estadual de Campinas, e-mail: \\ lucila@fec.unicamp.br , (4) Albert-Ludwigs-University Freiburg, e-mail: andreas.matzarakis@ meteo.uni- \\ freiburg.de
}

\section{RESUMO}

Este artigo visa avaliar a influência da geometria urbana no conforto térmico humano em cânions viários de Campinas. Foram selecionados dois cânions viários urbanos na orientação no eixo da rua leste-oeste, um em zona de baixa e outro em alta densidade de construção. Os dados meteorológicos coletados foram: temperatura do ar, umidade relativa do ar, velocidade do vento e radiação solar. Foram entrevistados 286 pedestres. Os resultados de campo foram comparados com as simulações a partir de modelos tridimensionais desenvolvidos no software RayMan Pro. O índice de conforto aplicado nesse estudo é Temperatura Fisiologicamente Equivalente (PET). Os resultados obtidos mostraram que a promoção do vento e o sombreamento de edifícios e vegetação dentro dos cânions viários foram capazes de mitigar não só a temperatura do ar, mas também o PET. Além disso, as sensações de conforto térmico aumentam à medida que velocidade do vento e o sombreamento aumentam no interior do cânion. Os pedestres mostraram uma preferência em andar na sombra durante o período quente e também a melhoria da sensação de conforto à medida que aumenta a velocidade do vento no interior do cânion. A quantificação do ambiente construído e simulação de cenários futuros a partir do software RayMan permite uma análise prática e rápida do ambiente térmico. Essa ferramenta pode ser muito útil para arquitetos, urbanistas e planejadores que buscam melhorar o conforto térmico em cidades tropicais.

Palavras-chave: temperatura fisiologicamente equivalente (PET), cânions urbanos, conforto térmico em ambientes externos.

\begin{abstract}
This paper main evaluated the influence of urban geometry in human thermal comfort of street canyons in Campinas by field measurements, users' interviews and computational simulations. Two street canyons in east-west direction and high and low density was selected. Meteorological data collected was air temperature, relative humidity, wind and solar radiation. It was interviewed 286 users. The results of field measurements were compared with simulations of tridimensional models using software RayMan Pro. The comfort index applied in this study is Physiologically Equivalent Temperature (PET). The results showed that wind and shade promote by trees and buildings were able to mitigate not only air temperature, but also PET. Additionally, thermal comfort increases as the wind speed and shade inside the street canyon. Pedestrians prefer walking in the shade during the warm period. The quantification of urban environment and futures scenarios simulations by RayMan allow practice and fast analyses of thermal environment. This toll can be used by architects, urban designers and planners that are worried to improve thermal comfort in tropical cities.
\end{abstract}

Keywords: Physiologically Equivalent Temperature (PET), urban street canyons, human thermal comfort in outdoor spaces. 


\section{INTRODUÇÃO}

O conforto térmico em ambientes abertos é influenciado pelo ambiente construído, como por exemplo, calor antropogênico (ICHINOSE et al., 1999), características dos materiais das superfícies, evaporação e evapotranspiração da vegetação (ROBITU et al., 2006), sombreamento arbóreo, abrigo ou edifícios (LIN et al., 2010) e velocidade do vento (PRATA, 2005; ABREU-HARBICH et al., 2014a). O conhecimento das condições climáticas em cânions urbanos através de medições de campo e simulações computacionais permite que projetistas e planejadores possam desenvolver espaços urbanos confortáveis e energeticamente eficientes.

Alguns estudos têm discutido o efeito do sombreamento em ambientes abertos a partir de estudos envolvendo características como a orientação da rua e relação altura e largura (H/W) (ALI-TOUDERT; MAYER, 2007; EMMANUEL et al., 2007; ABREUHARBICH et al.; 2014b) e o fator de visão do céu (FVC) (BOTTYAN; UNGER, 2003; GIUNTA et al., 2010). O sombreamento do ambiente construído por vegetação ou por edifícios podem melhorar o conforto térmico (LIN et al.; 2010, DACANAL et al, 2010). Porém, a permeabilidade do vento no ambiente construído pode melhorar ainda mais as sensações de conforto térmico em cidades tropicais como Santos e São Paulo (PRATA, 2005).

A sensação térmica varia de pessoa para pessoa em diferentes climas (GIVONI et al. 2003; CHENG; NG, 2006; NIKOLOPOULOU; LYKOUDIS, 2006; LIN; MATZARAKIS, 2008). Os residentes de Campinas estão acostumados ao clima quente e relativamente menos tolerantes às temperaturas frias (DACANAL et al., 2010). A quantificação do ambiente térmico dentro dos cânions viários utilizano o software RayMan não só permite a construção de um panorama bioclimatico urbano e simular situações futuras, mas estudar qual variável climática tem maior influencia no microclima urbano (FRÖHLICH; MATZARAKIS, 2013; ABREU-HARBICH et al., 2014b).

O objetivo desse artigo é avaliar a influencia da geometria urbana no conforto térmico humano em cânions viários de Campinas através de medições de campo, entrevistas com pedestres e simulações computacionais através do software RayMan Pro. Foram selecionados dois cânions viários representativos da cidade de Campinas em zonas de alta e baixa densidade de construção.

\section{METODOLOGIA}

\subsection{Clima de Campinas}

Campinas está localizada a $22^{\circ} 48^{\prime} 57^{\prime \prime} \mathrm{S}, 47^{\circ} 03^{\prime} 33^{\prime \prime} \mathrm{W}$ e com altitude de $640 \mathrm{~m}$. O clima da cidade é do tipo Cwa, segundo a classificação Köppen, ou seja, Tropical de Altitude, apresentando um verão quente e úmido e um inverno seco e frio (KOTTEK et al.; 2006). A média anual da temperatura do ar de $22,3{ }^{\circ} \mathrm{C}$, índice pluviométrico anual de $1411 \mathrm{~mm}$, com a predominância de chuvas nos meses de novembro a março e períodos de estiagem de 30 a 60 dias nos meses de julho e agosto. O período de verão, quando as temperaturas máximas variam entre $28,5{ }^{\circ} \mathrm{C}$ e $30,5{ }^{\circ} \mathrm{C}$, e as temperaturas mínimas variam entre $18,1^{\circ} \mathrm{C}$ e $19,9{ }^{\circ} \mathrm{C}$ correspondem aos meses de novembro a abril. O período de inverno, com temperaturas máximas variando entre $24,8{ }^{\circ} \mathrm{C}$ e $29,1{ }^{\circ} \mathrm{C}$ e as temperaturas mínimas entre $11,3{ }^{\circ} \mathrm{C}$ e $13,8{ }^{\circ} \mathrm{C}$ correspondem aos meses de junho, julho e agosto. 


\subsection{Locais de Medição}

Foram escolhidos dois cânions urbanos na cidade de Campinas para serem estudados, sendo esses em regiões de baixa e alta densidade de construção (Fig. 01). O primeiro local de estudo localiza-se em uma região de alta densidade, no bairro de uso misto, Cambuí. Compreende o trecho na Rua Conceição entre a Rua Boaventura do Amaral e Rua Padre Vieira, com aproximadamente $70 \mathrm{~m}$ de comprimento. A área apresenta largura de rua de $7,5 \mathrm{~m}$ e largura de calçada bastante variada ao longo do trecho estudado, variando de 1,5 a $3 \mathrm{~m}$. As edificações são de grande porte, com edifícios de até 15 pavimentos. A relação altura/largura do cânion é igual a 4 . O fator de visão do céu (FVC) é igual a 0.23 (Fig.1). A vegetação se limita praticamente aos pequenos jardins na frente dos edifícios e a 3 palmeiras Syagrus romanzoffiana e uma árvore de médio porte em uma das esquinas. A área apresenta um grande tráfego de pedestres e carros na região, diferente dos outros locais.

O segundo local de estudo localiza-se no bairro Taquaral e apresenta média densidade, situa-se na Rua Gilardi Vicente, com aproximadamente $100 \mathrm{~m}$ a partir da Rua Pé Manuel Bernardes. O trecho apresenta orientação do eixo da rua Leste-Oeste, largura de via de 7,5 m, com calçadas estreitas que variam em torno de $2 \mathrm{~m}$. Na composição do solo há o predomínio do asfalto na rua e pedras na calçada. As construções na região são predominantemente de um pavimento, com algumas de 2 pavimentos, a maioria com grades ou muros na frente e cobertura de telha cerâmica. A relação altura/largura do cânion é igual a 0.7. O fator de visão do céu (FVC) é igual a 0.58 (Fig. 1). Quanto à vegetação, a área apresenta poucas árvores, totalizando 8 árvores de pequeno a médio porte, cujas sombras não abrangem uma grande área e se limitam à região inferior à copa. As espécies predominantes são Lagerstroemia indica e Aroeira Salsa.

\section{Figura 1 - Localização dos cânions viários estudados}

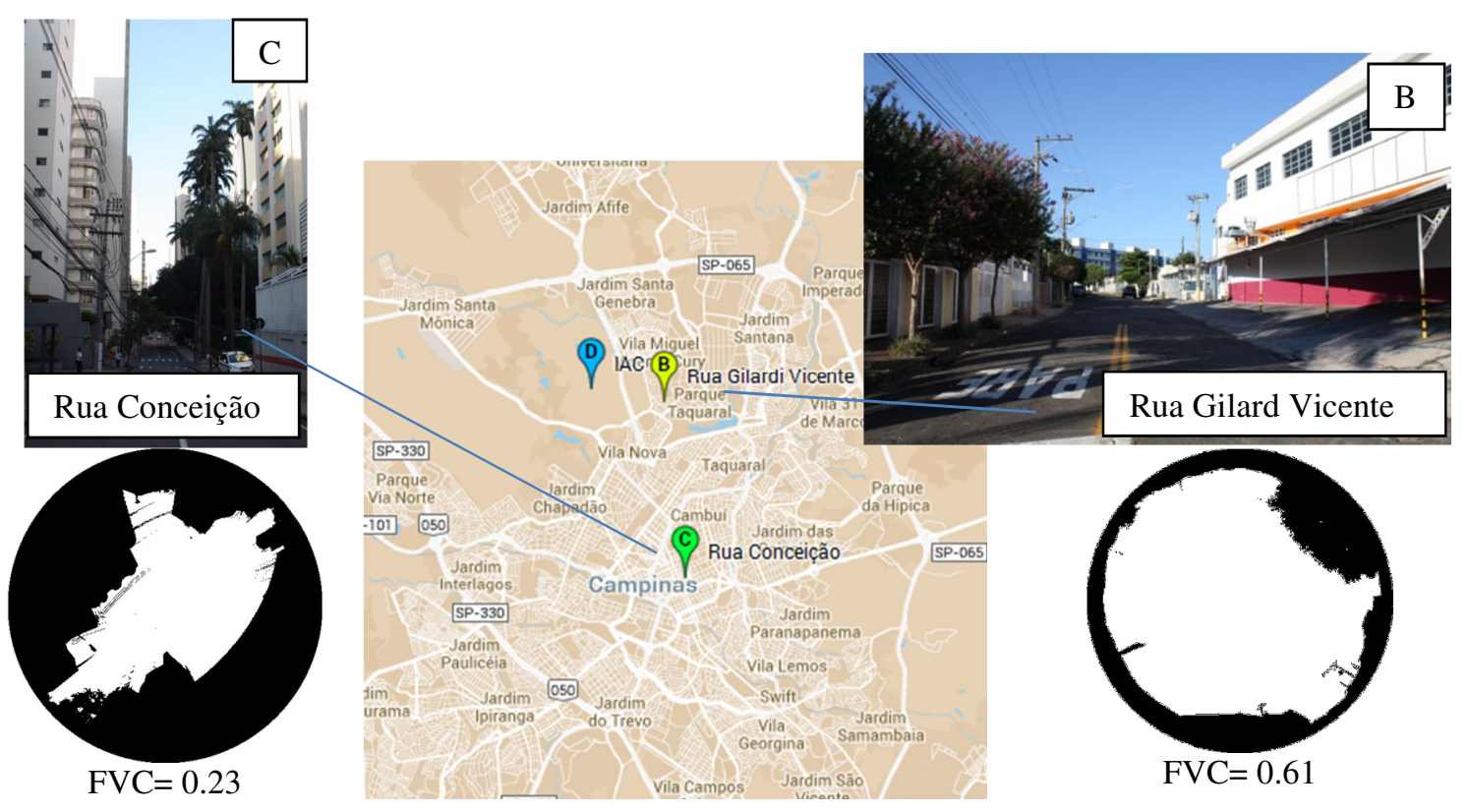

\subsection{Medições de Campo: Medidas Móveis}

A coleta de dados meteorológicos foi realizada durante um período de 10 horas durante um dia típico e representativo de verão em cada cânion selecionado. Foram 
estabelecidos para as análises seis pontos de medições, localizados na calçada face norte e sul do cânion, no caso de um cânion posicionado na orientação do eixo da rua LesteOeste (Fig. 2). A estação meteorológica muda de posição a cada 15 minutos.

Foram coletados: radiação solar, temperatura do ar, umidade relativa, velocidade do vento e temperatura de globo a partir de uma estação móvel (altura 1,5 m) constituída de um datalogger para registros de temperatura do ar e umidade relativa com protetores para a radiação (Testo 177-H1), datalogger com sonda externa para medição da temperatura de globo (Testo 175 T2), Net Radiômetro, com piranômetro e pirgeômetro (CNR 1-Kipp \& Zonen) e um anemômetro (Testo 445) (Fig. 3). Essa metodologia de coleta de dados se baseia em Correa et al. (2012) e Projeto RUROS (Rediscovering the Urban Realm and Open Spaces).

\section{Figura 2 - Localização dos cânions viários estudados}

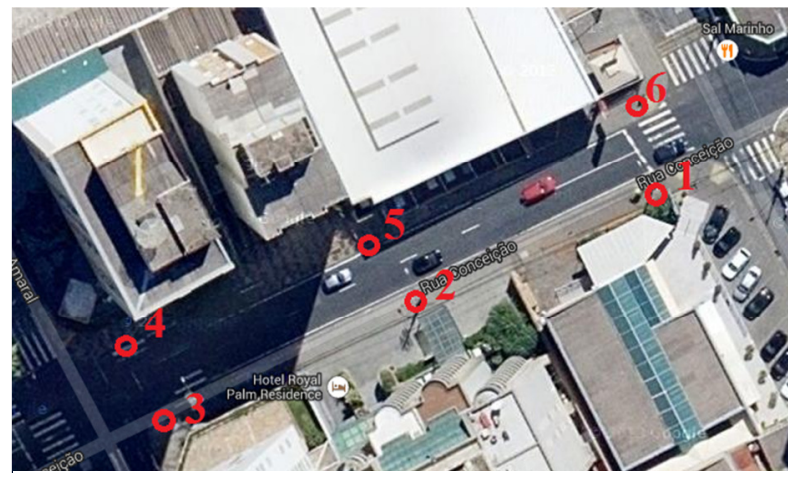

1- Rua Conceição, Cambuí

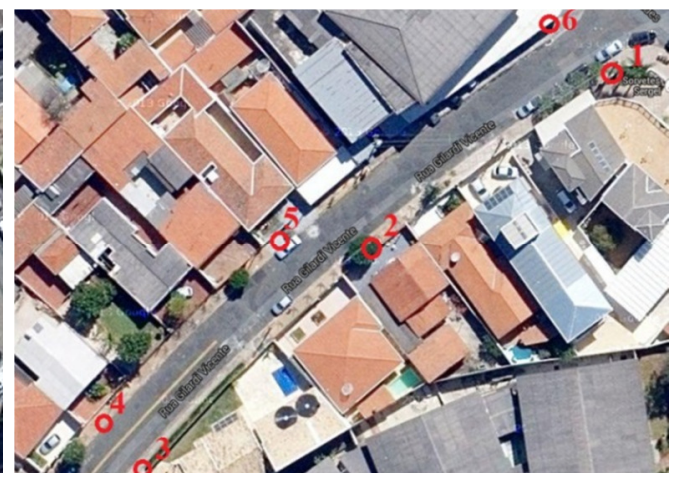

2- Rua Giliardi Vicente, Parque Taquaral

Figura 3 - Estação Meteorológica Móvel
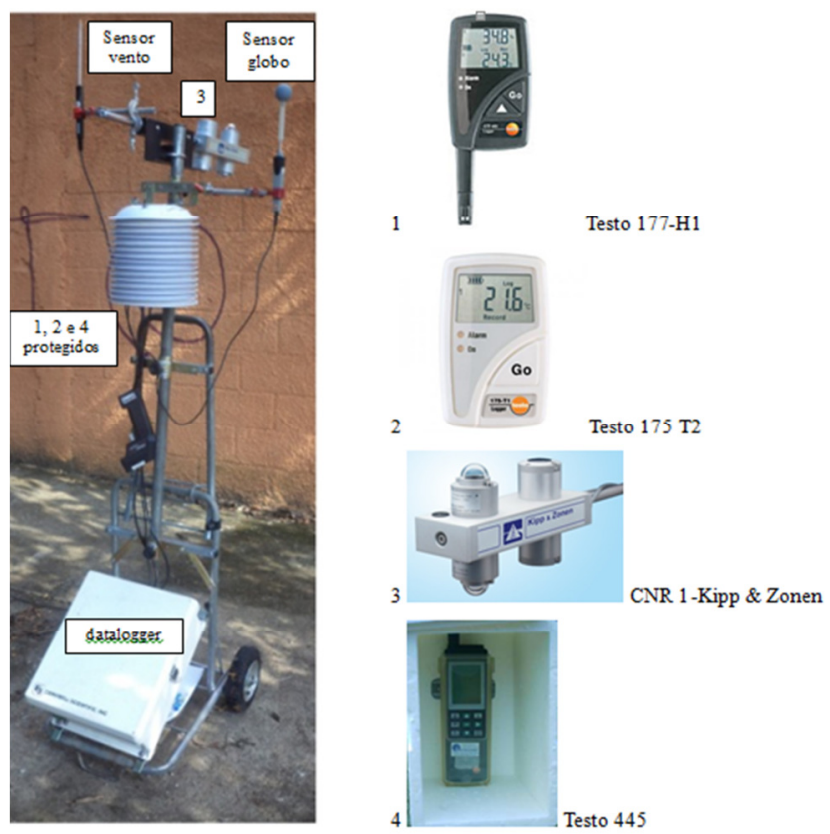

\subsection{Análises dos dados}

Vários índices integradores dos fatores térmicos do ambiente e do equilíbrio térmico do corpo humano têm sido aplicados para avaliar o conforto térmico em ambientes 
externos: PMV (Voto médio estimado) (FANGER, 1972), SET* (Temperatura Efetiva Padrão) (GAGGE et al., 1986) OUT_SET* (SPAGNOLO; DE DEAR, 2003) e PET (Temperatura Fisiológicamente Equivalente) (VDI, 1998). O PET permite a uma pessoa sem treinamento específico comparar os efeitos integrantes das mais complexas condições térmicas em ambientes externos com sua experiência em ambientes internos (HÖPPE, 1999) e torna os resultados mais compreensíveis para os planejadores urbanos ou regionais, que não estão tão familiarizados com a moderna terminologia de biometeorologia humana (MATZARAKIS, 2001). O PET é o índice adotado para avaliar o conforto térmico de seres humanos em espaços externos neste estudo. $\mathrm{O}$ software RayMan será empregado para calcular o (MATZARAKIS et al., 2007). Para comparar os resultados de índices como o Voto Médio Estimado (PMV) com o PET, Matzarakis e Mayer (1996) desenvolveram uma divisão em classes de sensações térmicas para os seres humanos e estresse por calor baseando em um gasto metabólico de $80 \mathrm{~W}$ e resistência térmica da roupa em 0.9 clo. Outras pesquisas fizeram ajustes da escala de sensações térmicas em diferentes regiões tropicais, tabela 1.

Tabela 1 Sensações Térmicas do PET para Europa, Taiwan e São Paulo

\begin{tabular}{|l|c|c|c|c|}
\hline $\begin{array}{c}\text { Sensação } \\
\text { Térmica }\end{array}$ & $\begin{array}{c}\text { PMV } \\
\text { (FANGER,1972) }\end{array}$ & $\begin{array}{c}\text { PETparaEuropean } \\
\text { ('CPET) } \\
\text { (MATZARAKIS; } \\
\text { MAYER,1996) }\end{array}$ & $\begin{array}{c}\text { PETpara Taiwan } \\
\text { ('CPET) } \\
\text { (UN;)MATZARAKIS, } \\
\text { 2008) }\end{array}$ & $\begin{array}{c}\text { PETparaSãoPaulo } \\
\text { ('CPET) } \\
\text { (MONIEIRO; } \\
\text { ALUCCI,2009) }\end{array}$ \\
\hline Muito Frio & $-3,5$ & $<4$ & $<14$ & \\
\hline Frio & $-2,5$ & $4-8$ & $14-18$ & $<4$ \\
\hline Fresco & $-1,5$ & $8-13$ & $18-22$ & $4-12$ \\
\hline Leve Frescor & $-0,5$ & $13-18$ & $22-26$ & $12-18$ \\
\hline Comfortavel & 0 & $18-23$ & $26-30$ & $18-26$ \\
\hline Leve Calor & 0,5 & $23-29$ & $30-34$ & $26-31$ \\
\hline Calor & 1,5 & $29-35$ & $34-38$ & $31-43$ \\
\hline Quente & 2,5 & $35-41$ & $38-42$ & $>43$ \\
\hline Muito Quente & 3,5 & $>41$ & $>42$ & \\
\hline
\end{tabular}

\subsection{Entrevistas com os Pedestres}

Foram aplicados 286 questionários durante a coleta de dados de campo, abordando-se os pedestres que passavam nas ruas sobre a sensação térmica e a preferencia do ambiente térmico. Na Rua Conceição, Cambuí, passaram pelos pontos de medição cerca de 1260 pessoas e 3550 carros, e na Rua Gilard Vicente, 133 pessoas e 232 carros. Apesar do grande número de pedestres, há uma dificuldade em entrevistar essas pessoas pois elas geralmente passam muito apressadas pelo experimento.

O perfil do entrevistado é constituído por jovens e adultos, entre 18 e 35 anos, sendo $42 \%$ de mulheres e $57 \%$ de homens. Todos estavam andando, cerca de $140 \mathrm{~W}$, e vestindo short e camiseta, cerca de 0.4 clo. Os entrevistados foram arguidos, em geral, sobre a sensação térmica e a preferência do ambiente térmico.

\subsection{Simulação com o Software RayMan}

Para analisar a influência da geometria urbana, as áreas estudadas foram modeladas no software RayMan Pro (Fig. 4) de acordo com as configurações urbanas dos locais de medição. Após a modelagem do ambiente real, o software permite calcular o PET para 
cada ponto analisado dentro do cânion viário utilizando os seguintes dados de entradas: temperatura do ar, umidade, velocidade do vento e radiação solar. Os valores da razão de Bowen e albedo foram adaptados para o local. Maiores informações podem ser encontradas em MATZARAKIS et al. (2007).

A fim de obter um resultado comparativo representativo, os dados meteorológicos, temperatura do ar e umidade relativa do ar, foram coletados simultaneamente nos jardins frontais das edificações localizadas nas áreas estudadas, por um período de 10 dias (18 a 28 de janeiro de 2014). Os dados de velocidade do vento e radiação solar foram obtidos através da estação meteorológica localizada no Instituto Agronômico de Campinas (IAC), $\left(22^{\circ} 54^{\prime} \mathrm{S}, 4^{\circ} 05^{\prime} \mathrm{W} ; 669 \mathrm{~m}\right)$ para o mesmo período. Segundo Matzarakis e Mayer (1996), a velocidade do vento foi corrigida para o local a partir da formula:

$$
\mathrm{WS}_{1.1}=\mathrm{WS}_{\mathrm{h}} *(1.1 / \mathrm{h})^{0.12 \mathrm{Zo}+0.18}
$$

onde, $\mathrm{WS}_{1.1}=$ velocidade do vento a $1.1 \mathrm{~m}$;

$\mathrm{WS}_{\mathrm{h}}=$ velocidade do vento medido na estação;

$\mathrm{h} \quad=$ altura da altitude medida em metros

Zo = rugosidade

Figura 4 - Estudos de sombras das ruas modelada no software

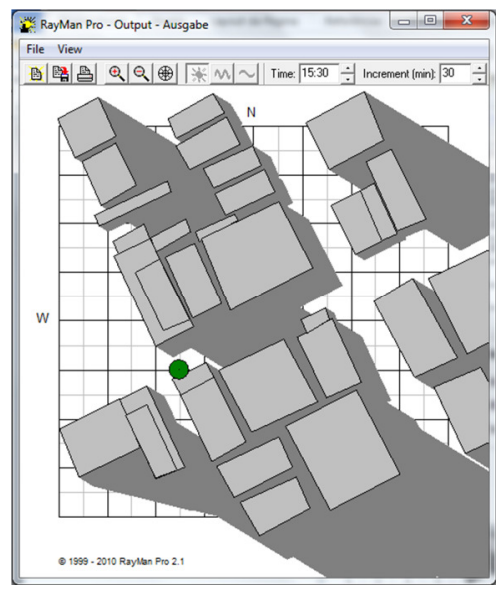

Rua Conceição, Cambuí

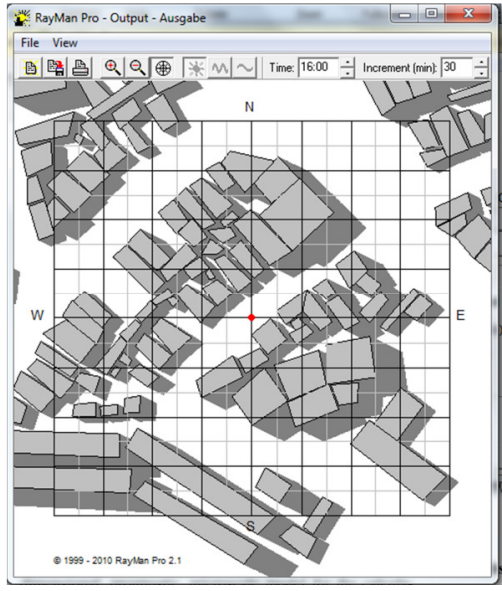

Rua Vicente Gilard, Taquaral

\section{RESULTADOS}

\subsection{Estudos de Campo}

A figura 5 apresenta os resultados de temperatura do ar (Ta), PET e velocidade do vento para as ruas Conceição, zona de alta densidade e Gilard Vicente, de baixa densidade. Observou-se que os valores do PET são influenciados pelo sombreamento e velocidade do vento nas duas ruas, pois à medida que a velocidade do vento aumenta ou o ponto de medição é submetido à sombra, os valores do PET diminuem.

Na Rua Conceição, durante o período de medição foram consideradas: $24 \%$ de Ta e $19 \%$ do PET como temperaturas confortáveis $\left(18^{\circ}\right.$ a $\left.23^{\circ} \mathrm{C}\right) ; 41 \%$ de Ta e $26 \%$ de PET como leve calor $\left(23^{\circ}\right.$ a $\left.29^{\circ} \mathrm{C}\right) ; 34 \%$ de Ta e $36 \%$ de PET como quente $\left(29^{\circ}\right.$ a $\left.35^{\circ} \mathrm{C}\right)$ e $14 \%$ de PET como estresse por calor (acima de $35^{\circ} \mathrm{C}$ ). Já na Rua Gilard Vicente, foram consideradas: $5 \%$ de Ta e $7 \%$ do PET como temperaturas confortáveis $\left(18^{\circ}\right.$ a $\left.23^{\circ} \mathrm{C}\right)$; 
$32 \%$ de Ta e $17 \%$ de PET como leve calor $\left(23^{\circ}\right.$ a $\left.29^{\circ} \mathrm{C}\right) ; 61 \%$ de Ta e $31 \%$ de PET como quente $\left(29^{\circ}\right.$ a $\left.35^{\circ} \mathrm{C}\right)$ e $5 \%$ de Ta e $46 \%$ de PET como estresse por calor (acima de $\left.35^{\circ} \mathrm{C}\right)$.

A figura 6 apresenta os resultados das entrevistas com os pedestres. $\mathrm{O}$ ambiente foi considerado: confortável por $25.9 \%$ dos homens e $23.8 \%$ das mulheres na Rua Conceição e $11.8 \%$ dos homens e $6.4 \%$ das mulheres na Rua Gilard Vicente; pouco quente por $27.6 \%$ dos homens e $19.3 \%$ das mulheres na Rua Conceição e $15.3 \%$ dos homens e $19.1 \%$ das mulheres na Rua Gilard Vicente; quente por $25.9 \%$ dos homens e $23.8 \%$ das mulheres na Rua Conceição e $11.8 \%$ dos homens e $6.4 \%$ das mulheres na Rua Gilard Vicente; muito quente por $29.3 \%$ dos homens e $26.3 \%$ das mulheres na Rua Conceição, e $51.8 \%$ do homens e $38.3 \%$ na Rua Gilard Vicente.

Figura 5 - Resultados das medidas de campo para as ruas estudadas

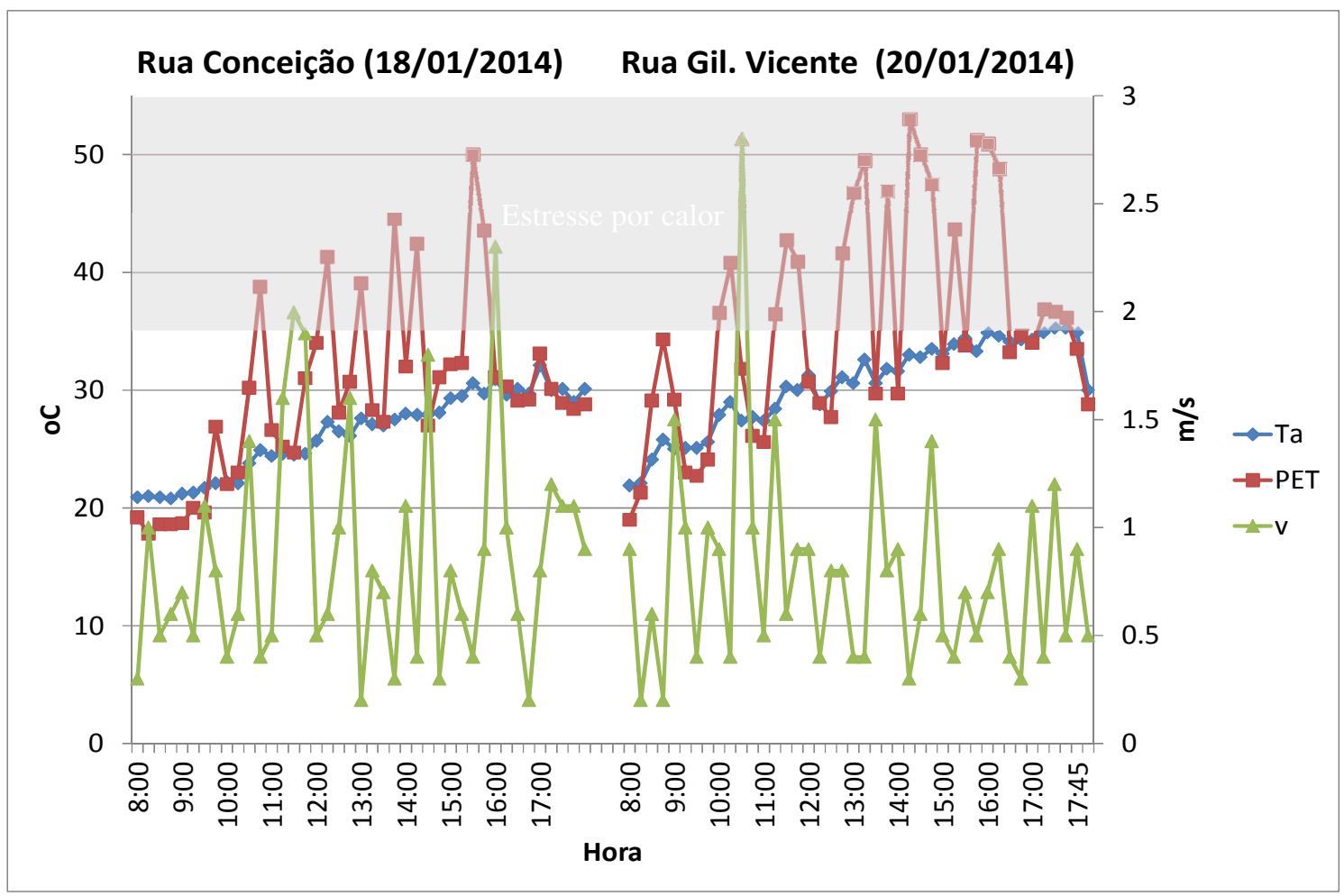

Figura 6 - Entrevista com os pedestres

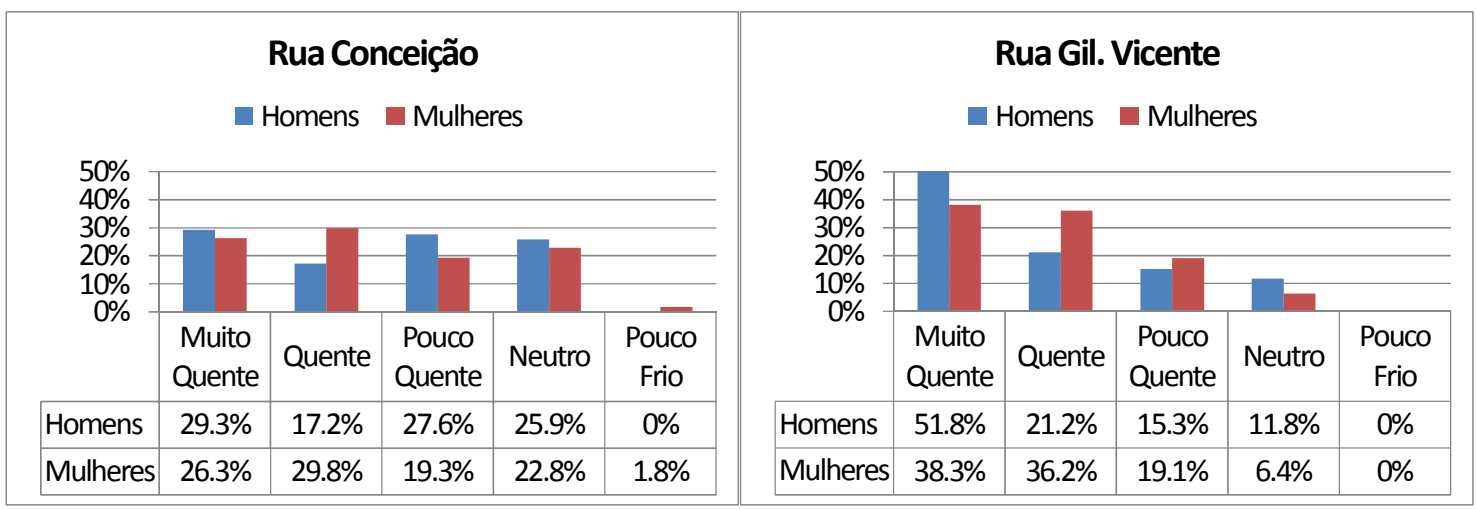




\subsection{Simulação}

A figura 7 apresenta os resultados da simulação de verão para um período de 10 dias para todos os pontos de medição nas duas ruas estudadas. Observou-se que as temperaturas do ar e PET na Rua Gilard Vicente são mais altas que na Rua Conceição durante o dia. Mas durante a noite, as temperaturas do ar e PET são mais altas na Rua Conceição do que na Rua Gilard Vicente. Esse fenômeno justifica a maior incidencia das ilhas de calor em zonas de alta densidade.

\section{Figura 7 - Estudos de sombras da Rua Gilard Vicente modelada no software}

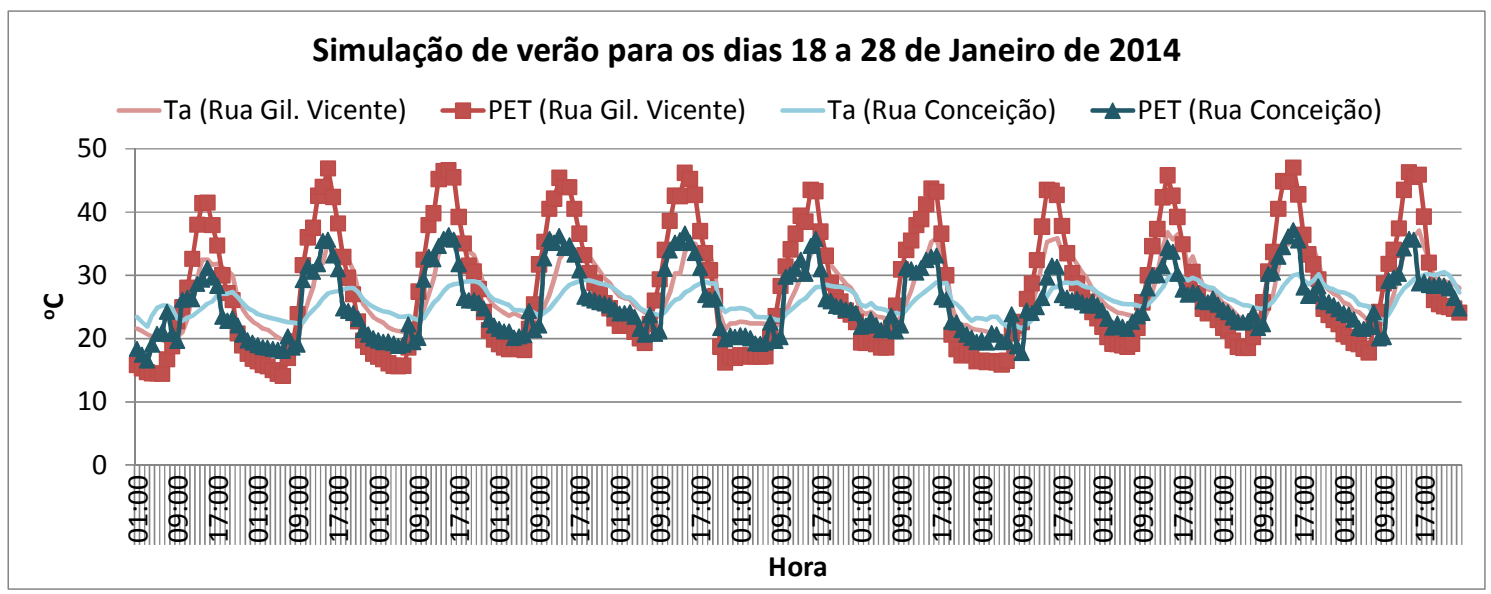

\section{CONCLUSÕES}

Este estudo analisou a influencia da geometria urbana no microclima de cânions viários, a partir de medições de campo e simulações computacionais utilizando o software RayMan Pro. A análise de cânions urbanos em zonas de baixa e alta densidade de construção pode ser quantificada através da aplicação do modelo Rayman (Matzarakis et al, 2007; 2010).

Este estudo sugere que a promoção de sombra e vento nas cidades é capaz de melhorar o conforto térmico em cidades tropicais, confirmando os resultados de Lin et al. (2010) e Abreu-Harbich et al. (2014a; 2014b). O plantio estratégico de árvores poderia trabalhar como um elemento mitigador das ilhas de calor nas cidades tanto em zonas de alta como em zonas de baixa densidade de construção. Entretanto, o melhor posicionamento da vegetação pode ser estudado a partir do software RayMan para a criação de futuros cenários.

Neste estudo, a situação atual pode ser modelada rapidamente e quantificada com o uso o software RayMan Além disso, o software permite a construção de um panorama bioclimático baseado em dados históricos. Em um trabalho futuro, novos cenários poderão ser simulados visando à melhoria térmica dentro desses cânions viários. A simulação de cenários futuros a partir do software RayMan pode auxiliar arquitetos, urbanistas e planejadores na tomada de decisões de projetos ou planejamento urbano. A correta aplicação dessa ferramenta permitiria a arquitetos e planejadores a desenvolver diretrizes que pudessem construir espaços urbanos cada vez mais adaptados às mudanças climáticas e consequentemente, trazer mais bem-estar a população. 


\section{AGRADECIMENTOS}

Ao CNPq, DAAD e CAPES pelo apoio financeiro no desenvolvimento da pesquisa, FAPESP, processo $n^{0}$ 2014/16362-1, pelo apoio na divulgação da pesquisa e também aos técnicos Obadias P. da Silva e Daniel Celente, do Laboratório de Conforto Ambiental e Física da Faculdade de Engenharia Civil, Arquitetura e Urbanismo da UNICAMP.

\section{REFERENCIAS}

ABREU-HARBICH, L. V., LABAKI, L.C., MATZARAKIS, A. Thermal Bioclimate as factor in urban and architectural planning in tropical climates - The case of Campinas, Brazil. Urban Ecosystems, v. 17, n. 2, p. 489-500, Jun. 2014a.

ABREU-HARBICH, L. V., LABAKI, L.C., MATZARAKIS, A. Thermal bioclimate in idealized urban street canyons in Campinas, Brazil. Theoretical and Applied Climatology, v.115, p.333-340, Jan. 2014b.

ALI-TOUDERT, F.; MAYER, H., Thermal comfort in an east-west oriented street canyon in Freiburg (Germany) under hot summer conditions. Theoretical and Applied Climatology, v. 87, n. 1-4, p. 223-237. Jan. 2007.

BOTTYAN, Z., UNGER,J. A multiple linear statistical model for estimating the mean maximum urban heat island. Theoretical and Applied Climatology. v. 75,n.3-4, p. 233-243. Set. 2003

CORREA, E., RUIZ, M. A. , CANTON, A. , LESINO, G. Thermal comfort in forested urban canyons of low building density. An assessment for the city of Mendoza, Argentina. Building and Environment, v. 58, p.219-230, Dec. 2012.

CHENG, V., NG, E. Thermal comfort in urban open spaces for Hong Kong. Architectural Science Review, v. 49, p. 236-242, Jun. 2006.

DACANAL, C., LABAKI, L. C. SANTOS, T.M.L. Vamos passear na floresta! O conforto térmico em fragmentos florestais urbanos. Ambiente Construído, v. 10, n. 2 , 2010 .

EMMANUEL, R., ROSENLUND, H., JOHANSSON, E. Urban shading - a design option for the tropics? A study in Colombo, Sri Lanka. International Journal of Climatology. v. 27, n. 14 p.1995-2004, Nov. 2007.

FANGER, P. O. Thermal comfort. Analysis and applications in environmental engineering. McGraw-Hill, New York. p. 244, 1972

FRÖHLICH, D., MATZARAKIS, A. Modeling of Changes in Thermal Bioclimate: Examples Based on Urban Spaces in Freiburg, Germany. v.111, n.3-4, p. 547-558, Fev. 2013.

GAGGE, A. P., FOBELETS, E L., BERGLUND, G. A standard predictive index of human response to the thermal environment. ASHRAE Trans 92(pt 2B), p. 709-731, 1986.

GIVONI, B., NOGUCHI, M., SAARONI, H., POCHTER, O., YAACOV, Y., FELLER, N., BECKER, S. Outdoor comfort research issues. Energy and Buildings, v. 35, n. 1, p.77-86, Jan. 2003. 
GIUNTA, M. B. ; TENTE, C.M. ; NAKATA, C. M. ; SOUZA, L. C. L. Fator de visão do céu e intensidades de ilhas de calor na escala do pedestre. Ambiente Construído (Online), v. 10, p. 155-167, Out./Dez. 2010.

HÖPPE, P.R. Heat balance modelling. Experientia, v. 49, n.9, p. 741-746, 1993.

ICHINOSE, T., SHIMODOZONO, K. , HANAKI, K. Impact of anthropogenic heat on urban climate in Tokyo. Atmospheric Environment, v. 33, n. 24-25, p. 3897-3909, Out. 1999.

KOTTEK, M., GRIESER, J. , BECK, C. , RUDOLF, B. , RUBEL, F. World Map of the Köppen-Geiger climate classification updated. Meteorologische Zeitschrift, v. 15, n.3,p. 259-263, Jun. 2006

LIN, T. P.; MATZARAKIS, A. Tourism climate and thermal comfort in Sun Moon Lake, Taiwan. Int. J. Biometeorology, v. 52, n.4, p.281-290, mar. 2008.

LIN, T., MATZARAKIS, A.; HWANG, R. Shading effect on long-term outdoor thermal comfort, Building and Environment. Building and Environment, v.45, n. 1, p. 213-221, Jan. 2010.

MATZARAKIS, A., MAYER ,H. Another kind of environmental stress: thermal stress. WHO Collaborating Centre for Air Quality Management and Air Pollution Control Newsletters, v.18, p.7-10, 1996.

MATZARAKIS, A. Die thermische Komponente des Stadtklimas. Ber. Meteorol. Inst. Univ. Freiburg Nr. 6. 2001.

MATZARAKIS, A., RUTZ, F. , MAYER, H. Modelling Radiation fluxes in simple and complex environments - Application of the RayMan model. International Journal of Biometeorology, v. 51, p. 323-334, 2007.

MATZARAKIS, A., RUTZ, F. , MAYER, H. Modelling Radiation fluxes in simple and complex environments - Basics of the RayMan model. International Journal of Biometeorology, v. 54, p. 131-139, 2010.

MONTEIRO, L. M., ALUCCI, M. P. Proposal of an outdoor thermal comfort index for subtropical urban areas. Proceedings of 3rd International Conference on Passive and Low Energy Cooling for the Built Environment, Rhodes. 29 set. - 1 out. 2010.

NIKOLOPOULOU, M., LYKOUDIS, S. Thermal comfort in outdoor urban spaces: Analysis across different European countries. Building and Environment, v. 41, n.11, p. 1455-1470, Nov. 2006.

PRATA, A. R. Impacto da altura dos edifícios nas condições de ventilação natural do meio urbano. 2005. 243 f. Tese (Doutorado em Arquitetura e Urbanismo, Estruturas Ambientais Urbanas) Faculdade de Arquitetura e Urbanismo, Universidade de São Paulo, São Paulo, 2005.

ROBITU, M., MUSY, M., INARD, C., GROLEAU, D. Modeling the influence of vegetation and water pond on urban microclimate. Solar Energy, v. 80, n.4, p. 435-447, Abr. 2006

SPAGNOLO, J. C., DE DEAR, R. J. A Human Thermal Climatology of Subtropical Sydney. International Journal of Climatology, v. 23, n.11, p. 1383-1395, Set. 2003.

VDI: Methods for the human-biometerological assessment of climate and air hygiene for urban and regional planning. Part I: Climate, VDI guideline 3787. Part 2. Beuth.1998. 